\title{
No-recoil approximation to knock-on exchange potential in the double folding model for heavy-ion collisions
}

\author{
K. Hagino, T. Takehi, and N. Takigawa \\ Department of Physics, Tohoku University, Sendai 980-8578, Japan
}

(Dated: October 25, 2018)

\begin{abstract}
We propose the no-recoil approximation, which is valid for heavy systems, for a double folding nucleus-nucleus potential. With this approximation, the non-local knock-on exchange contribution becomes a local form. We discuss the applicability of this approximation for the elastic scattering of ${ }^{6} \mathrm{Li}+{ }^{40} \mathrm{Ca}$ system. We find that, for this system and heavier, the no-recoil approximation works as good as another widely used local approximation which employs a local plane wave for the relative motion between the colliding nuclei. We also compare the results of the no-recoil calculations with those of the zero-range approximation often used to handle the knock-on exchange effect.
\end{abstract}

PACS numbers: 24.10.-i,25.70.Bc,21.60.-n

The double folding model has been widely used to describe the real part of optical potential for heavy-ion collisions [1, 2, 3]. The direct part of the double folding potential is constructed by convoluting an effective nucleonnucleon interaction with the ground state density distributions of the projectile and target nuclei. In the double folding model, the exchange contribution originating from the antisymmetrization of the total wave function of the system is customarily taken into account simply through the single nucleon knock-on exchange term. The exchange term leads to a non-local potential. Since it is cumbersome to handle the resultant integro-differential equation, a local approximation has usually been employed. In the past, many calculations have been performed along this line by introducing a pseudo zero-range nucleon-nucleon interaction to mock up the knock-on exchange effect 1, 2, 4]. The strength of the pseudo interaction has been tuned so as to reproduce exact results of the integro-differential equation for proton scattering from various target nuclei at several incident energies 4]. This approach, in conjunction with the (density dependent) Michigan-three-range Yukawa (M3Y) interaction [5, 6], has successfully accounted for observed elastic and inelastic scattering for many colliding systems 1, 2].

Recently, a more consistent treatment for the exchange term has also been considered [3, 7, 8, 9, 10]. This approach obtains a local potential by employing a local approximation to the momentum operator (local momentum approximation) [11, 12]. Since the local momentum depends explicitly on the potential itself, there arises the self-consistency problem, which however can be solved iteratively. Since the exchange potential is directly constructed from a given nucleon-nucleon interaction of finite range, this approach is more favorable than the zero range approximation. In fact, the finite range treatment for the exchange term has enjoyed a success in reproducing the experimental angular distributions for light heavy-ion scattering where the zero range approximation fails $7,8,13]$.

Despite its success, however, there is a potential difficulty in this approach. That is, the iterative procedure for the self-consistent problem may not work in the clas- sically forbidden region, where the local momentum is imaginary. Although the frozen density approximation used in the double folding model could be questionable at these low energies, one may still attempt to construct a nucleus-nucleus potential with the double folding procedure.

The aim of this paper is to propose an alternative local approximation to the knock-on exchange term in the double folding model, which is applicable even in the classically forbidden region. To this end, we exploit the fact that the non-locality of the exchange potential arises from the recoil effect and thus its range is not large for heavy systems 2, 14]. We simply ignore the recoil effect (i.e., introduce the no-recoil approximation), and obtain a local nucleus-nucleus potential. As in the local momentum approximation, the only ingredients needed in our approach are a nucleon-nucleon interaction and the one-body density matrices of the colliding nuclei. Our approach is thus complementary to the local momentum approximation, which is valid even for light systems but may not work at very low energies, especially at energies below the Coulomb barrier. A similar no-recoil approximation has been discussed in Refs. 14, 15], as well as for heavy-ion transfer reactions in Ref. 16]. Here, we systematically investigate the applicability of the no-recoil approximation by comparing to the exact result as well as to the result in the local momentum approximation.

We begin with the Schrödinger equation based on the double folding model for the relative motion between the colliding nuclei,

$\left[-\frac{\hbar^{2}}{2 \mu} \nabla^{2}+V_{d}(r)+V_{C}(r)-i W(r)-E\right] \psi(\boldsymbol{r})+\left[V_{\mathrm{ex}} \psi\right](\boldsymbol{r})=0$,

where $\mu$ and $V_{C}$ are the reduced mass and the Coulomb potential, respectively, and $-i W$ is the imaginary potential which simulates the inelastic and fusion processes. $V_{d}$ is the direct contribution of the double folding potential given by [1, 2]

$$
V_{d}(r)=\int d \boldsymbol{r}_{P} d \boldsymbol{r}_{T} \rho_{P}\left(\boldsymbol{r}_{P}\right) \rho_{T}\left(\boldsymbol{r}_{T}\right) v\left(\boldsymbol{r}_{T}-\boldsymbol{r}_{P}-\boldsymbol{r}\right),
$$


while the exchange part is given by 17

$$
\begin{aligned}
{\left[V_{\mathrm{ex}} \psi\right](\boldsymbol{r})=} & \int d \boldsymbol{r}_{P} d \boldsymbol{r}_{T} \rho_{T}\left(\boldsymbol{r}_{T}-\boldsymbol{s}, \boldsymbol{r}_{T}\right) \rho_{P}\left(\boldsymbol{r}_{P}+\boldsymbol{s}, \boldsymbol{r}_{P}\right) \\
& \times v(\boldsymbol{s}) \psi(\boldsymbol{r}+\alpha \boldsymbol{s}),
\end{aligned}
$$

where $\boldsymbol{s}=\boldsymbol{r}_{T}-\boldsymbol{r}_{P}-\boldsymbol{r}$ and $\alpha=\left(A_{P}+A_{T}\right) / A_{P} A_{T}=$ $1 / A_{P}+1 / A_{T}$. Here, $v(\boldsymbol{s})$ is an effective nucleon-nucleon interaction. $\rho_{T}$ and $\rho_{P}$ are the one-body density matrix for the target and projectile nuclei, respectively, and $\rho_{i}(\boldsymbol{r})$ in Eq. (2) is their diagonal component ( $i=\mathrm{P}$ or T). In order to evaluate those density matrices, we use the local density approximation [8, 17, 18],

$$
\rho(\overline{\boldsymbol{r}}+\boldsymbol{s} / 2, \overline{\boldsymbol{r}}-\boldsymbol{s} / 2) \sim \rho(\overline{\boldsymbol{r}}) \hat{j}_{1}\left(k_{F}(\overline{\boldsymbol{r}}) s\right),
$$

where $\hat{j}_{1}(x)=3(\sin x-x \cos x) / x^{3}$, and evaluate the local Fermi momentum $k_{F}(\boldsymbol{r})$ in the extended Thomas-Fermi approximation.

One can obtain a local approximation to Eq. (3) by noticing

$$
\psi(\boldsymbol{r}+\alpha \boldsymbol{s})=e^{i \alpha \boldsymbol{s} \cdot \hat{\boldsymbol{p}} / \hbar} \psi(\boldsymbol{r}),
$$

and evaluating the momentum operator $\hat{\boldsymbol{p}}$ in the local WKB approximation $\boldsymbol{k}(r) \hbar$ (local momentum approximation [3, 7, 8, 9, 10, 11, 12]). This yields a local exchange potential

$$
\left[V_{\mathrm{ex}} \psi\right](\boldsymbol{r})=V_{\mathrm{ex}}(r) \psi(\boldsymbol{r})
$$

with

$$
\begin{aligned}
V_{\mathrm{ex}}(r)= & \int d \boldsymbol{r}_{P} d \boldsymbol{r}_{T} \rho_{T}\left(\boldsymbol{r}_{T}-\boldsymbol{s}, \boldsymbol{r}_{T}\right) \rho_{P}\left(\boldsymbol{r}_{P}+\boldsymbol{s}, \boldsymbol{r}_{P}\right) \\
& \times v(\boldsymbol{s}) e^{i \alpha \boldsymbol{k}(r) \cdot \boldsymbol{s}}
\end{aligned}
$$

where the magnitude of the local momentum is given by

$$
k(r)=\sqrt{\frac{2 \mu}{\hbar^{2}}\left[E-V_{d}(r)-V_{C}(r)-V_{\mathrm{ex}}(r)\right]} .
$$

Notice that the local momentum $k(r)$ has to be determined consistently to the exchange potential $V_{\mathrm{ex}}$, as it appears both on the right and left hand sides of Eq. (7). One can also obtain the same expression for the exchange potential (7) by constructing the trivially equivalent local potential for Eq. (3) and approximating the relative wave functions with those in the WKB approximation, that is, $\psi(\boldsymbol{r}) \sim e^{i \boldsymbol{k}(r) \cdot \boldsymbol{r}} / \sqrt{k(r)}$.

A further simplification for the exchange term can be achieved for heavy systems. To this end, we remark that $\alpha$ in Eq. (3) arises from the variation of center of mass as a consequence of the exchange of nucleons between the projectile and target nuclei. It is nothing more than the recoil effect due to the nucleon exchange [2, 14], and may be neglected for heavy systems. For instance, the value of $\alpha$ is 0.0673 and 0.192 for ${ }^{16} \mathrm{O}+{ }^{208} \mathrm{~Pb}$ and ${ }^{6} \mathrm{Li}+{ }^{40} \mathrm{Ca}$, respectively. If one neglects $\alpha$ in Eqs. (3) or (7), the exchange potential $V_{\mathrm{ex}}$ is simply given by,

$$
V_{\mathrm{ex}}(r)=\int d \boldsymbol{r}_{P} d \boldsymbol{r}_{T} \rho_{T}\left(\boldsymbol{r}_{T}-\boldsymbol{s}, \boldsymbol{r}_{T}\right) \rho_{P}\left(\boldsymbol{r}_{P}+\boldsymbol{s}, \boldsymbol{r}_{P}\right) v(\boldsymbol{s}) .
$$

We call this approximation the no-recoil approximation. Notice that the self-consistency problem is not involved in this approximation, in contrast to the local momentum approximation.

Let us now investigate numerically the applicability of the no-recoil approximation. For this purpose, we choose the ${ }^{6} \mathrm{Li}+{ }^{40} \mathrm{Ca}$ system. We use a version of the densitydependent M3Y (DDM3Y) interaction, CDM3Y6 [9] as the nucleon-nucleon interaction, $v$. It is given by

$$
v(r)=F_{d}(\rho)\left[11061.625 \frac{e^{-4 r}}{4 r}-2537.5 \frac{e^{-2.5 r}}{2.5 r}\right]
$$

for the direct part, (2), and

$$
\begin{aligned}
v(r)= & F_{\mathrm{ex}}(\rho)\left[-1524.25 \frac{e^{-4 r}}{4 r}-518.75 \frac{e^{-2.5 r}}{2.5 r}\right. \\
& \left.-7.8474 \frac{e^{-0.7072 r}}{0.7072 r}\right] \quad(\mathrm{MeV}),
\end{aligned}
$$

for the exchange part, (3). Here, the length is in the unit of $\mathrm{fm}$, and the density dependent strength is given by

$$
F(\rho)=C\left[1+\alpha e^{-\beta \rho}-\gamma \rho\right]
$$

with $\rho=\rho_{P}\left(\boldsymbol{r}_{P}\right)+\rho_{T}\left(\boldsymbol{r}_{T}\right)$ and $\rho=\rho_{P}\left(\boldsymbol{r}_{P}+\boldsymbol{s} / 2\right)+$ $\rho_{T}\left(\boldsymbol{r}_{T}-\boldsymbol{s} / 2\right)$ for the direct and the exchange contributions, $F_{d}$ and $F_{\text {ex }}$, respectively. The value for $C, \alpha, \beta$, and $\gamma$ can be found in Ref. [9]. We assume that the imaginary potential $W$ is proportional to the double folding potential with the knock-on exchange term estimated in the zero-range approximation. For the projectile and target densities, we use the same densities as in Refs. [1] and [19], respectively. The normalization factor is set to be unity for all the calculations reported below. The barrier height thus obtained is $8.44 \mathrm{MeV}$ in the no-recoil approximation, while it is $8.45,8.44$, and $8.50 \mathrm{MeV}$ at $E_{\text {lab }}=30$, 50.6 , and $156 \mathrm{MeV}$, respectively, in the local momentum approximation.

Figure 1 shows the angular distribution of elastic ${ }^{6} \mathrm{Li}$ $+{ }^{40} \mathrm{Ca}$ scattering at $E_{\mathrm{lab}}=156.0,50.6$ and $30.0 \mathrm{MeV}$ as indicated in the figure. The filled circles are the exact results of the integro-differential equation, which fully retains the non-locality of the exchange potential. The solid and dashed lines are obtained in the present norecoil approximation and in the local momentum approximations, respectively. For comparison, the figure also shows the results of the zero-range approximation (see the dotted line), that is obtained by replacing the nucleon-nucleon potential for the exchange term given by Eq. (11) with

$$
v(\boldsymbol{r})=F_{\mathrm{ex}}(\rho) \cdot J(E) \delta(\boldsymbol{r})
$$




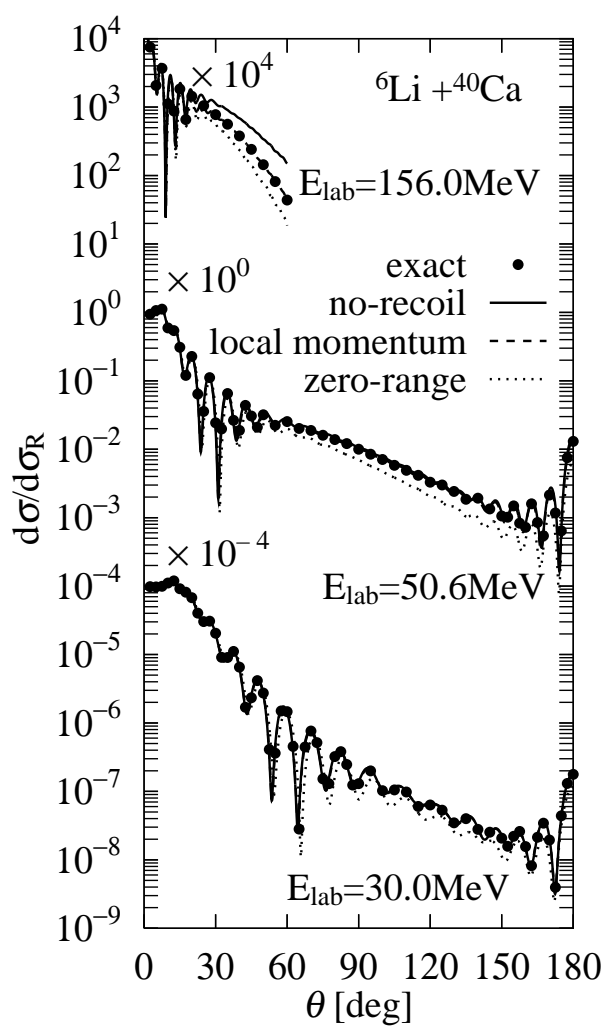

FIG. 1: The angular distribution of elastic ${ }^{6} \mathrm{Li}+{ }^{40} \mathrm{Ca}$ scattering at $E_{\text {lab }}=156.0,50.6$ and $30.0 \mathrm{MeV}$ obtained with several methods. The filled circles are the exact results of the integro-differential equation with the full non-local potential. The solid, the dashed, and the dotted lines are obtained in the no-recoil, the local momentum, and the zero-range approximations, respectively.

with $J(E)=-590\left(1-0.002 E_{\mathrm{lab}} / A_{P}\right)\left(\mathrm{MeV} \cdot \mathrm{fm}^{3}\right)$ [1, 2, 4]. The strength was tailored particularly for the proton scattering, but has been used for heavy-ion scattering as well by introducing the dependence on the incident energy per projectile nucleon.

The figure shows that the no-recoil approximation leads to similar results as the local momentum approximation for this system at the lowest two energies. They well reproduce the exact results. We have confirmed that this is the case also for heavier system such as ${ }^{16} \mathrm{O}+$ ${ }^{208} \mathrm{~Pb}$, as it is expected. In contrast, we observe significant difference between the exact results and the results of the zero range approximation.

At the highest energy, $E_{\text {lab }}=156.0 \mathrm{MeV}$, the no-recoil approximation does not work well. This is due to that the local momentum $k(r)$ is relatively large at this high energy, and the exponent in Eq. (17) cannot be neglected even if the value of $\alpha$ itself is small. For a lighter system, $\alpha+{ }^{90} \mathrm{Zr}$, where $\alpha=0.261$ and the barrier height is around 11.7 MeV, we find that the no-recoil approximation does not work even at $E_{\text {lab }}=40.0 \mathrm{MeV}$. It is thus clear that, in order for the no-recoil approximation to work well, the inverse reduced mass $\alpha$ needs to be small and at the same time the bombarding energy has to be relatively low.

In summary, we proposed the no-recoil approximation for the double folding model. It neglects the recoil effect due to the knock-on exchange of nucleons between the projectile and target nuclei. The resultant exchange potential has a simple local form. We examined its applicability for heavy-ion reactions by studying the angular distribution of elastic ${ }^{6} \mathrm{Li}+{ }^{40} \mathrm{Ca}$ scattering. We found that the no-recoil approximation reproduces reasonably well the exact results with the full non-local exchange potential, and works as good as the local momentum approximation for this system unless the bombarding energy is much above the Coulomb barrier. The performance of the no-recoil approximation improves for heavier systems. The zero range approximation, one the other hand, does not reproduce well the results of original nonlocal potential. Since the no-recoil approximation does not involve the iterative procedure, it is much simpler than the local momentum approximation. We thus advocate the use of no-recoil approximation in analyzing heavy-ion scattering.

In the double folding model, the exchange effect has been conventionally taken into account only through the knock-on exchange term. This is reasonable for peripheral collisions, since the knock-on exchange has the longest range among other exchange terms [20]. However, it is not obvious at all whether other exchange terms are negligible when the potential in the inner region plays a role, such as in rainbow scattering or in fusion reactions. In this connection, we mention that a similar idea as in the no-recoil approximation proposed in this paper enables us to follow the idea of the resonating group method (RGM), that fully incorporates the exchange effect, relatively easily even for heavy systems (see Ref. 21] for an early attempt). We will report on such studies in a separate paper 22].

We thank T. Wada for useful discussions, and the Australian National University for their warm hospitality where this work was completed. We also thank discussions with the members of the Japan-Australia Cooperative Scientific Program "Dynamics of Nuclear Fusion: Evolution Through a Complex Multi-Dimensional Landscape". This work was supported by the Grant-in-Aid for Scientific Research, Contract No. 16740139 from the Japanese Ministry of Education, Culture, Sports, Science, and Technology. 
(1997).

[3] D.T. Khoa and G.R. Satchler, Nucl. Phys. A668, 3 (2000).

[4] W.G. Love and L.W. Owen, Nucl. Phys. A239, 74 (1975).

[5] G. Bertsch, J. Borysowicz, H. McManus, and W.G. Love, Nucl. Phys. A284, 399 (1977).

[6] A.M. Kobos, B.A. Brown, R. Lindsay, and G.R. Satchler, Nucl. Phys. A425, 205 (1984).

[7] D.T. Khoa, Nucl. Phys. A484, 376 (1988).

[8] D.T. Khoa, W. von Oertzen, and H.G. Bohlen, Phys. Rev. C49, 1652 (1994).

[9] D.T. Khoa, G.R. Satchler, and W. von Oertzen, Phys. Rev. C56, 954 (1997).

[10] D.T. Khoa, Phys. Rev. C63, 034007 (2001).

[11] B. Sinha, Phys. Rep. 20, 1 (1975).

[12] B. Sinha and S.A. Moszkowski, Phys. Lett. 81B, 289 (1979).

[13] M. Katsuma, Y. Sakuragi, S. Okabe, and Y. Kondo, Prog. Theo. Phys. 107, 377 (2002).
[14] M. Golin, F. Petrovich, and D. Robson, Phys. Lett. 64B, 253 (1976).

[15] T. Kaneko, M. LeMere, and Y.C. Tang, Phys. Rev. C44, 1588 (1991); T. Kaneko and Y.C. Tang, Prog. Theo. Phys. 101, 591 (1999).

[16] R.A. Broglia and A. Winther, Heavy Ion Reactions, Vol. 84 in Frontiers in Physics Lecture Note Series (AddisonWesley, Redwood City, CA, 1991).

[17] W.G. Love, Nucl. Phys. A312, 160 (1978).

[18] X. Campi and A. Bouyssy, Phys. Lett. 73B, 263 (1978).

[19] M. El-Azab Farid and G.R. Satchler, Nucl. Phys. A438, 525 (1985).

[20] K. Aoki and H. Horiuchi, Prog. Theo. Phys. 68, 2028 (1982); H. Horiuchi, in Proceedings of the fourth Int. Conf., "Clustering Aspects of Nuclear Structure and Nuclear Reactions", Chester, 1984, eds. J.S. Lilley and M.A. Nagarajan, p. 35.

[21] H. Friedrich, Nucl. Phys. A224, 537 (1974).

[22] T. Takehi, N. Takigawa, and K. Hagino, to be published. 